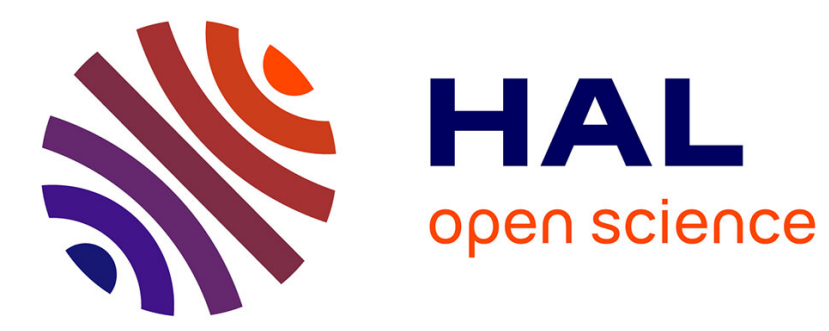

\title{
MRI Strain Imaging of the Carotid Artery: Present Limitations and Future Challenges
}

\author{
Aart Nederveen, Stéphane Avril, Lambert Speelman
}

\section{To cite this version:}

Aart Nederveen, Stéphane Avril, Lambert Speelman. MRI Strain Imaging of the Carotid Artery: Present Limitations and Future Challenges. Journal of Biomechanics, 2014, 47 (4), pp.824 - 833. 10.1016/j.jbiomech.2014.01.014 . hal-01024645

\section{HAL Id: hal-01024645 \\ https://hal.science/hal-01024645}

Submitted on 16 Jul 2014

HAL is a multi-disciplinary open access archive for the deposit and dissemination of scientific research documents, whether they are published or not. The documents may come from teaching and research institutions in France or abroad, or from public or private research centers.
L'archive ouverte pluridisciplinaire HAL, est destinée au dépôt et à la diffusion de documents scientifiques de niveau recherche, publiés ou non, émanant des établissements d'enseignement et de recherche français ou étrangers, des laboratoires publics ou privés. 


\title{
MRI Strain Imaging of the Carotid Artery: Present Limitations and Future Challenges
}

\author{
Aart J. Nederveen ${ }^{1}$, Stéphane Avril ${ }^{2}$, Lambert Speelman ${ }^{3}$ \\ ${ }^{1}$ Department of Radiology, Academic Medical Center Amsterdam, the Netherlands \\ ${ }^{2}$ Center for Biomedical and Healthcare Engineering, \\ Ecole Nationale Supérieure des Mines de Saint-Étienne, France \\ ${ }^{3}$ Department of Biomedical Engineering, Erasmus MC Rotterdam, the Netherlands
}

Corresponding author:

Aart J. Nederveen, PhD

Department of Radiology

Academic Medical Center

University of Amsterdam

Meibergdreef 9

1105 AZ Amsterdam, the Netherlands

Telephone: +31 (0) 205664975

Word count abstract: 203

Word count manuscript (introduction through discussion): 6580

References: 52

Figures: 6

Tables: 1

Keywords: carotid artery plaque, strain, cine MRI, phase contrast MRI, non-rigid image registration 


\begin{abstract}
Rupture of atherosclerotic plaques in the carotid artery is a main cause of stroke. Current diagnostics are not sufficient to identify all rupture-prone plaques, and studies have shown that biomechanical factors improve current plaque risk assessment. Strain imaging may be a valuable contribution to this risk assessment. MRI is a versatile imaging technique that offers various methods that are capable of measuring tissue strain. In this review, MR imaging techniques using conventional cine MRI and displacement (DENSE) or velocity (PC MRI) encoding are discussed, together with post-processing techniques based on time-resolved MRI data. Although several MRI techniques are being developed to improve time-resolved $M R$ imaging, current technical limitations related to spatial and temporal resolutions render MRI strain imaging currently unfit for carotid plaque strain evaluation.
\end{abstract}

A novel approach using non-rigid image registration of MR images to determine strain in carotid arteries based on black blood cine MRI is proposed in this review. This and other post-processing techniques based on time-resolved MRI data may provide a good estimate of plaque strain, but are also dependent on the spatial and temporal resolution of the MR images. However, they seem to be the most promising approach for MRI based plaque strain analysis in the near future. 


\section{Introduction}

Atherosclerosis is a cardiovascular disease, characterized by lipid accumulation in the arterial wall, which is separated from the blood by a fibrous cap (Slager et al., 2005). Rupture of these caps of plaques in carotid arteries leads to stroke in the majority of cases (Casscells et al., 2003; Golledge et al., 2000). Carotid artery plaques can be surgically removed to reduce risk on stroke. Physicians nowadays judge the risk of plaque rupture mainly on general risk factors, like hypertension and blood cholesterol levels (D'Agostino et al., 2001) and the amount of lumen narrowing caused by the plaque (Halliday et al., 2004). However, these risk factors are not sufficient to identify all high risk plaques. Better risk assessment of carotid plaque rupture is therefore highly desired to reduce the incidence of stroke and plaque rupture-related mortality.

Biomechanical studies showed that stresses in the cap, induced by the blood pressure, provide a better rupture risk assessment (Makris et al., 2010; Sadat et al., 2011). Unfortunately, biomechanical analysis of plaques is subject to modeling assumptions, like simplified material models, unknown boundary conditions and geometry inaccuracies. Additionally, creating biomechanical models is very labor intensive, time consuming, and requires knowledge of the used methods, which hampers the introduction of biomechanical plaque analysis in daily clinical practice. Alternatively, cap strain, a measure for blood pressure induced cap deformation, is strongly related to the cap stress and can potentially be visualized with non-invasive dynamic imaging. Cap strain is not only determined by cap thickness and stiffness, but also by the geometry and characteristics of other plaque components, like a lipid pool or calcifications (Akyildiz et al., 2011). Carotid artery strain imaging may therefore be a valuable contribution to the clinical determination of plaque rupture risk. Additionally, strain imaging may provide data on the mechanical properties of the different plaque components, for future biomechanical analyses. Previous studies in coronary and femoral arteries showed with invasive 
ultrasound measurements that strain values can indeed serve as a surrogate marker for plaque composition and vulnerability (Schaar et al., 2006; Naim et al., 2013).

Although intravascular ultrasound strain imaging is a promising method that may be used to detect vulnerable plaques in the future, the invasive nature and limited penetration depth of this catheterbased technique currently render this tool unsuitable for clinical carotid plaque evaluation. Also, ultrasound can, at this moment, not provide 3D time dependent imaging data and is a limited imaging modality for highly calcified plaques, which are often found in carotid arteries. Alternative techniques to obtain local carotid plaque strain information based on transcutaneous ultrasound are only just being developed and at this point suffer from a low sensitivity, especially for highly calcified plaques (Hansen et al., 2009; Schaar et al., 2003).

Magnetic resonance imaging (MRI) is the most versatile imaging method for characterizing the atherosclerotic disease process and is available in both clinical and research settings. It can provide volumetric imaging data and its usefulness for measuring strain has extensively been shown for the myocardium (Tee et al., 2013) and the aorta (Merkx et al., 2009; van't Veer et al., 2008; Wedding et al., 2002). This review briefly introduces some basic concepts of MRI and discusses current MRI techniques in conjunction with several post-processing techniques that may be used to evaluate carotid artery vessel wall and plaque strain. Additionally, a new approach to obtain strain data from dynamic MRI data is proposed, illustrated with pilot data. Conclusions are presented on the current possibilities and limitations of measuring carotid artery strain with MR imaging. We will discuss future requirements and developments of MR strain imaging for carotid artery plaques. 


\section{Basic concepts of MRI}

Nuclear magnetic resonance emerged as an imaging technique in the 1980s (Bernstein et al., 2004). Key to the understanding of the physical mechanisms behind MRI is the behaviour of hydrogen atoms in a strong magnetic field $\mathrm{B}_{0}$. Clinical MRI scanners typically have $\mathrm{B}_{0}$ field strengths of 1.5 Tesla or 3.0 Tesla. The nucleus of a hydrogen atom contains one proton. This proton carries a physical property, called spin, which can be represented by a vector, and in many aspects, resembles a very small compass needle. Under the influence of a strong external magnetic field the spin vectors on average tend to align with the main magnetic field. The summation of all spin vectors in a certain area is called the net magnetisation vector. The magnitude of this magnetisation vector can be recorded with MRI for each voxel, i.e. volume element, in a tissue. Since hydrogen atoms in biological tissue are most abundant in the form of water molecules, the main contribution to the MRI signal originates from the water in the human body during an MRI scan.

RF pulses

Application of a radiofrequency (RF) wave can strongly influence the dynamic behaviour of the net magnetisation vector. During application of an RF wave, the magnetisation vector in a volume rotates away from its initial main magnetic field direction. For many applications, the RF wave is switched off at the moment the magnetisation vector reaches the transverse plane (i.e. the plane perpendicular to the main magnetic field). In clinical practice, various MRI protocols are used, which apply a combination of RF pulses with different flip angles (FA), specifying the amount of rotation of the magnetisation vector.

After an RF pulse, the magnetisation vector (in part) resides in the transverse plane and precesses in this plane; i.e. it rotates around its main axis with the same frequency as the RF pulse, called the Larmor frequency. This frequency is defined by the gyromagnetic ratio $\gamma$, which is a physical constant that depends on the imaged hydrogen nuclei, multiplied with the strength of the main magnetic field $\left(B_{0}\right)$. At 
1.5 Tesla, the Larmor frequency equals $64 \mathrm{MHz}$. The precessional rotation of the magnetisation vector can be detected by a receiver MRI coil that is placed near the patient's body, as the precessing spins generate an electrical induction current in the coil. Eventually this current can be reconstructed into an MR image. After the RF pulse, the spins will recover to the main magnetic field direction. The time required for this recovery is represented by the time constant $\mathrm{T} 1$. The time constants $\mathrm{T} 2$ and $\mathrm{T} 2$ * represent the decrease in current in the coil, caused by dephasing of spin vectors in tissue; a process that is called transverse relaxation. The time constants $\mathrm{T} 1, \mathrm{~T} 2$ and $\mathrm{T} 2^{*}$ are tissue specific and can be used to create contrast between different tissues.

\section{Spatial encoding}

During an MRI scan, spatial encoding is applied to differentiate between signals coming from different locations. The hardware components used in this process are called gradient coils. Gradient coils are located inside the MRI scanner and they effectuate linear changes in the main magnetic field along any direction inside the scanner. The strength of this gradient $G$ is specified in $\mathrm{mT} / \mathrm{m}$ and indicates the slope of this linear change. Using this modulation of $\mathrm{B}_{0}$, a frequency can be attributed to a specific location, given the fact that the Larmor frequency is proportional to the local magnetic field $\mathrm{B}_{0}$. The frequency at which the precessing spins rotate differs per location. The magnitude of the signals for different frequencies is related to the proton densities at the specific locations. If the spin density is high, a high amplitude of the RF wave is generated at that location. This process of spatial encoding is implemented in modern MRI scanners by Fourier transformation of the MR signals after the acquisition. The signals recorded with the coil reside in the time domain, also known as k-space, whereas after Fourier transformation the data are in the image domain. Data in k-space are acquired as complex numbers; consequently the MR image can be represented as magnitude and phase data. The acquisition of k-space data can be either slice-based or volume-based, resulting in either 2D or 3D k-space data. In many MRI 
sequences the time between the acquisition of consecutive portions of the k-space is called the repetition time (TR). Commonly, in one TR, one line in k-space is acquired after the application of one single RF pulse.

With the concepts outlined above one can appreciate the basic building block of an MRI sequence: an RF pulse directly followed by a readout gradient for spatial encoding. This building block is also denoted as gradient echo, referring to the peak of the MR-signal that is recorded at the echo time TE (see Figure 1A). Although in reality MRI pulse sequences carry much more complexity, this building block can be used to illustrate many of the MRI concepts that are being exploited in strain imaging.

In an MRI sequence based on gradient echoes, the largest portion of the TR is dedicated to the readout time for one k-line: $T_{a c q}$. This is identical to the number of time points $N$ at which the MRI signal is sampled, multiplied by the sampling time needed for each point, the latter being identical to the bandwidth of the receiver. In MRI, the product of the readout gradient strength $G$, the readout time $T_{a c a}$ and the voxel size $\Delta x$ is constant for a fixed field of view:

$$
G \cdot T_{a c q} \cdot \Delta x=\frac{4 \pi}{\gamma}
$$

From this equation, one important limitation of MRI concerning spatial resolution can be understood. In human MRI scanners the maximal gradient strength that can be used is limited by hardware and safety restraints. Decreasing the voxel size $\Delta x$ for a fixed field of view can therefore only be achieved if the readout time $T_{\text {acq }}$ increases, thus increasing the TR of the gradient echo. In time resolved MRI, the MRI sequence is synchronized with the heart cycle and the TR represents the temporal resolution of the sequence. In this case a higher spatial resolution leads inevitably to a lower temporal resolution of the MRI scan, and the other way around. 


\section{MRI imaging techniques for strain analysis}

Time resolved MRI can be used to determine tissue displacement. Strain MRI has mainly been developed for cardiac MRI. Several sophisticated imaging protocols were designed and thoroughly validated over the last decade (Tee et al., 2013; Wang and Amini, 2012). Direct translation of these methods to the carotid artery is hindered by several issues. For the carotid artery, the spatial resolution should be below $0.5 \mathrm{~mm}$, assuming that the mean wall thickness in patients with carotid artery disease is larger than 1 $\mathrm{mm}$ and that at least one voxel should always entirely reside in the vessel wall (van Wijk et al., 2013). Simply increasing the spatial resolution is difficult because the signal-to-noise ratio (SNR) decreases for smaller voxel sizes. To increase the SNR, dedicated carotid artery coils are required (Balu et al., 2009). Additionally, increasing the spatial resolution also results in a decrease in temporal resolution. In cardiac MRI, temporal resolutions are between $20-30 \mathrm{~ms}$ and ideally these should also be available for carotid MRI to avoid temporal blurring of the pulsatile motion patterns.

Table 1 briefly reviews and compares different techniques that have been used in the literature to measure vessel wall strain. The different techniques are discussed below. For each technique we will explain what is measured, how it works, and its potential to measure strain in the carotid artery. In some cases, MRI data will be shown for educational purposes, acquired in healthy volunteers on a 3T MRI scanner (Philips Healthcare, Best, The Netherlands), equipped with a dedicated 8 channel carotid coil. Informed consent was obtained.

\section{Cine MRI}

The workhorse for MR carotid artery strain imaging is cine MRI. Cine MRI is capable of imaging the movement of the carotid artery over time. Cine MRI is a straightforward MR acquisition protocol, consisting of repeated application of the gradient echo building block (see figure 1C). This pulse sequence can be either prospectively or retrospectively synchronized with the heart rhythm (Slavin and 
Bluemke, 2005). The temporal resolution of cine MRI is limited by the repetition time (TR) of the sequence. Completion of the sequence occurs when all k-lines needed for image reconstruction of the different heart phases are acquired. Using typical gradient strengths and bandwidths available at modern scanners, TRs are 3-5 ms at $2 \mathrm{~mm}$ spatial resolution and as large as $20 \mathrm{~ms}$ at $0.2 \mathrm{~mm}$ spatial resolution.

Cine MRI was used in several papers as a starting point for post-processing to derive tissue properties of carotid arteries (Avril et al., 2011a, 2011b; Franquet et al., 2013a, 2013b). Reported temporal resolutions were in the range of $20-50 \mathrm{~ms}$ in combination with spatial resolutions around $0.6 \mathrm{~mm}$. A disadvantage of conventional cine MRI is that only the lumen boundary is visible, whereas the vessel wall is hardly discernible, due to the limited spatial resolution and SNR of the wall compared to the lumen (see figure 2).

To improve the visibility of the lumen wall, one could apply blood suppression (black blood) techniques. In this case in between the RF pulses, extra pulses are added to suppress the flowing blood. In general, black blood techniques are difficult to combine with cine MRI and will always lower the temporal resolution of the sequence. Recently, a method for acquiring black blood cine MRI at high isotropic spatial resolution has been put forward by Koktzoglou et al. (2013), though the temporal resolution of this method was limited to $60 \mathrm{~ms}$ (Koktzoglou, 2013).

Increasing the lumen-wall contrast can be performed by using another gradient echo technique, called balanced SSFP. This technique is standard for cardiac cine imaging and has been used for strain measurements in the aorta (Herment et al., 2011). However, due to technical difficulties to apply this sequence at higher resolution, balanced SSFP has not been applied for carotid arteries so far. In balanced SSFP, banding artifacts that render the anatomy invisibly, scale with the TR of the sequence. Since a higher resolution will increase the TR, the resulting image quality will in general be inferior to cine MRI. 
In cardiac MRI, cine imaging is often combined with tagging, also called SPAtial Modulation Magnetization (SPAMM) (Young et al., 1993). In this approach, extra RF pulses are applied each heart beat that spatially modulate the magnetization within the selected slice to be imaged. In that case the cine gradient echo sequence registers the moving magnetization. Typically, a grid is used for spatial magnetization modulation. The resolution of the grid is much lower than the imaging resolution, hindering straightforward application of this method to the carotid artery, since the imaging resolution itself is already in the same range as the spatial extent of the features that should be visualized.

\section{Cine phase contrast MRI}

In cine phase contrast (PC) MRI (Lotz et al., 2002), phase images are used to calculate the velocity of the tissue inside a voxel. This technique is mainly applied for measuring blood flow velocity, but may also be used to measure the velocity of the vessel wall.

Cine PC MRI is based on the phenomenon that the displacement of hydrogen nuclei (protons or spins) in tissue, moving along a magnetic field gradient, translates in the phase data of the MR image. To this end, extra velocity-encoding gradients are added to a gradient echo sequence, comprising of two lobes of equal area and opposite polarity, a so-called bipolar gradient (Bernstein et al., 2004). To avoid phase contributions from other sources, a second acquisition is performed with an inverted bipolar gradient (toggling) (see figure 1D). After subtraction of the phase data, the remaining phase difference is proportional to the velocity of the tissue:

$$
\Delta \varphi=2 \gamma \cdot G \cdot v \cdot \delta^{2}
$$

Here, $\gamma$ is the gyromagnetic ratio, $\mathrm{G}$ is the gradient strength, $\delta$ is the gradient duration (see figure 1D) and $v$ is the velocity of the tissue. One should realize that the magnitude of the phase difference depends on both the velocity and the gradient strength, i.e. a low velocity can still result in a considerable phase 
difference if the gradient strength is sufficiently high. In this context, a velocity encoding parameter VENC is defined as $\pi /\left(2 \gamma \cdot G \cdot \delta^{2}\right)$, indicating the velocity for which the phase is equal to $\pi$.

As schematically illustrated in figure 1D, the temporal resolution in cine PC MRI is decreased compared to conventional cine MRI for two reasons: the addition of the bipolar gradients and the need for 2 TRs to measure the velocity in one direction.

Cine PC MRI is commonly used to measure blood flow, but also other physiological motion processes in the human body can be studied, like pulsatile motion of the vessel wall. The applied VENC should then be as low as $1-5 \mathrm{~cm} / \mathrm{s}$ in order to obtain enough velocity-to-noise-ratio at low velocities. Application of low VENCS requires relatively large gradient moments which can often only be reached by prolonged gradient duration, since the gradient strength itself is hardware limited. This again will decrease the temporal resolution (see figure 1E). One attractive advantage of low VENC values is that these gradients will suppress signal from flowing blood inside the vessel due to a phenomenon called intravoxel dephasing, that is described in more detail elsewhere (Nguyen et al., 2008).

In literature, some attempts have been made to measure the velocity of the aorta by using cine PCMRI, both in humans (Wedding et al., 2002) and in animals (Draney et al., 2004, 2002). Temporal and spatial resolutions were in the range of $50-60 \mathrm{~ms}$ and $0.3-0.8 \mathrm{~mm}$ respectively and VENCs to encode radial pulsatile motion were all below $5 \mathrm{~cm} / \mathrm{s}$. Figure 3 shows cine PC MRI images of a healthy subject with velocity encoding in the caudal-cranial direction (VENC $1 \mathrm{~cm} / \mathrm{s}$ ) for two different time points. In the phase difference images, the carotid artery wall clearly shows negative velocities in the top image, indicating a downward motion, and positive velocities in the bottom image, indicating an upward motion. Unfortunately, radial motion could not be captured due to the limited spatial and temporal resolution of $0.5 \mathrm{~mm}$ and $50 \mathrm{~ms}$. The tradeoff between spatial and temporal resolution is much more difficult than in the aorta, given the hardware limitations of the current MRI scanners. A minimum in- 
plane resolution of $0.5 \mathrm{~mm}$ is required to visualize the vessel wall, whereas temporal resolutions around 50 ms will already substantially mask important pulsatile motion patterns.

DENSE

A modification of the cine PC MRI technique is Displacement Encoding using Stimulated Echoes (DENSE) (Aletras et al., 1999). Instead of measuring velocity, DENSE can be used to measure tissue displacement.

In cine PC MRI it is possible to evaluate the velocity of tissue. Extrapolating this information to displacement involves integration over several heart phases and will be prone to errors, e.g. offsets in the velocity data and inaccurate determination of the maximal tissue displacement because of the limited temporal resolution of cine PC MRI at low VENCs (Lotz et al., 2002). Increasing the time interval between the lobes of the bipolar gradient would in principle allow for the encoding of motion over a much longer time period, thus incorporating the integration over time in the MRI sequence itself. This is performed in DENSE by making use of stimulated echoes that store the tissue magnetization temporarily along the longitudinal direction to avoid signal dephasing during the time period in between the gradient lobes. In DENSE, the accumulated phase can be expressed as the displacement of the tissue during this time period, which can be chosen such that wall displacement from systole to diastole can be captured.

It has been shown that from an MRI physics point of view, DENSE resembles the application of a SPAMM pre-pulse as explained above (Kuijer et al., 2006). However, in DENSE, the resolution of the tagging grid is no longer a limiting factor for measuring strain in structures smaller in size than the tag resolution. Lin and co-workers showed that DENSE derived strain was comparable to strain obtained from lumen circumference measurements from cine MRI (Lin et al., 2008). To our knowledge, DENSE has not been applied frequently in the carotid artery. Apart from resolution demands (at least $0.5 \mathrm{~mm}$ in-plane resolution), DENSE is not standardly available on clinical scanners, which explains its limited clinical application. 
Besides velocity (PC MRI) and displacement (DENSE) encoding techniques, strain encoding (SENC) MRI was developed to immediately encode the strain in the acquired images, without measuring the displacement or velocity first (Osman, 2003). However, SENC only provides strain data in the throughplane of the images and cannot be used for in-plane radial strain imaging.

\section{MR Elastography}

Strain measurements in the carotid arteries may also benefit from the growing field of MR elastography (MRE). With MRE, the vessel wall stiffness can be estimated from the MRI data.

In MRE a mechanical excitation at a frequency of $50-100 \mathrm{~Hz}$ is applied, inducing periodic tissue motion. MRI is far too slow to record this motion in real time. However, due to the periodicity of the applied motion and its fixed frequency, one can sample the motion consecutively at several positions during the motion cycle, using motion encoding gradients with varying time offsets, very similar to the bipolar gradients that are used in PC MRI. This will result in phase images that encode the displacement of the tissue during the motion cycle, generated by the propagating shear wave caused by the external vibration. The wavelength of the shear wave relates to the tissue stiffness (Bohte et al., 2013; Huwart et al., 2008).

MRE has mainly been applied in the liver to measure fibrosis, although some researchers recently applied this technique for aortic wall strain measurements (Kolipaka et al., 2011; Xu et al., 2013). Wave propagation was studied in the aorta and stiffness values were derived. The authors assume that when the aorta is vibrated, the aortic wall and the adjacent blood vibrate with the same frequency. This means that in their approach, the shear wave was measured in the aorta and not in the vessel wall itself.

So far, no literature was found that applies MRE in carotid arteries, but in principle the approach used in the aorta can be translated to the carotid artery. One can either measure the shear wave in the lumen or 
in the vessel wall itself. The latter will only be possible if sufficient spatial resolution is available, since the same spatial resolution restraints apply as for cine MRI. MRE may provide local variations in material parameters, for instance when imaging atherosclerotic plaques with different plaque components. Typically, in MRE, the tissue is assumed to behave as a linear elastic, isotropic material (Xu et al., 2013). In general, accounting for nonlinear of anisotropic tissue properties would result in more unknown parameters that should be determined from the MRE phase data and consequently imposes higher demands on the quality of the data. Although we know that biological material is generally non-linear and anisotropic, linearity may be assumed for the physiological pressure range. 


\section{MRI post-processing techniques for strain analysis}

As illustrated in the previous section, several techniques exist to derive strain or stiffness information of carotid arteries from MRI data. Local strain or stiffness values can be determined directly with displacement encoding (DENSE), velocity encoding (cine PC MRI), or strain encoding (SENC) MRI techniques, or with MRE, but none of these techniques has currently been proven to be robust for strain analysis in the carotids, mainly because of limited spatial and temporal resolution. However, postprocessing techniques based on cine MRI data, on which blood appears either bright (bright blood MRI) or dark (black blood MRI), may also provide local strain or stiffness data. Below, we review key publications illustrating the strengths and limitations of strain measurements based on bright and black blood cine MRI.

\section{Bright blood analysis}

Bright blood cine MRI sequences are available on all modern MRI scanners and do not impose heavy constraints on the spatial resolution as long as only the lumen contour needs to be segmented. Using a bright blood MRI sequence (see figure 2), the lumen contour or surface area over the heart cycle can be tracked and the relative difference in length or area between the systolic and dia stolic images can be computed. Only a global average circumferential strain can be derived using this method and variation in strain over the vessel circumference due to eccentric plaques cannot be captured.

Avril et al. (2011) developed a technique for sub-pixel local tracking of the vessel wall of the human common carotid artery imaged with bright blood MRI, with the purpose to derive the local elastic properties of the carotid artery (Avril et al., 2011a). Sub-pixel tracking of the lumen was achieved by applying the optical flow method (Beauchemin and Barron, 1995) on bright blood cine PC MRI magnitude images. With this method, the location of the artery wall is tracked over time. Radial displacements of the vessel wall are obtained and combined with pressure measurements to estimate the circumferential 
stress and strain in the arterial wall over time. The wall stiffness can then be estimated as the ratio between stress and strain, averaged over time. Feasibility of this method has been proven with healthy carotid artery measurements (Avril et al., 2011b).

Another method to identify the mechanical properties of arteries from bright blood cine PC MRI was introduced by Franquet et al. (2013a) (Franquet et al., 2013a). This method is based on minimizing the cost function that measures the distance between a template image and a registered image. From the cine PC MRI images (bright blood), diastolic and systolic images were chosen. The diastolic images were used to create a finite element (FE) model, with an initial estimation of material parameters. The computed displacement field was applied to the diastolic image to generate a registered image. The distance between the registered image and the phase contrast MR image at systole was minimized using a Levenberg-Marquardt algorithm, by iteratively updating the material parameters in the FE model.

Franquet et al. (2013a) validated this approach on a silicone phantom (Franquet et al., 2013a). In another paper by the same group, this approach was used to characterize the properties of human common carotid arteries (Franquet et al., 2013b). For this method, precise measurement of the blood pressure is crucial, as well as an accurate description of the tissues surrounding the artery. The average Young's moduli of the carotid wall and of the surrounding tissue were used as material properties. They showed significant differences between healthy and atherosclerotic patients (see figure 4). They also used images of the carotid arteries at different pressure levels throughout the cardiac cycle and showed that the Young's moduli of the wall was on average lower for low pressures, than for higher pressures. Carotid arteries seem to become stiffer with increasing age (figure 4), although a large variation within the age groups was observed. Especially in the highest age group, the variation is very large, as this group represents data from both diseased and non-diseased arteries. Although so far only the average stiffness of carotid arteries was determined, this method has the potential of identifying local mechanical 
properties of carotid artery plaque tissue, when the different components are modeled separately in the FE model. However, with an increasing number of plaque components of which the material properties need to be estimated, the number of optimal combinations of material properties may increase too. By defining additional boundary conditions on the finite element model and evaluating multiple (strainrelated) parameters, an optimal combination of material properties for the plaque components may be determined.

\section{Black blood analysis}

Compared to bright blood sequences, black blood sequences in general have a lower temporal resolution, as the suppression of signal from the blood requires additional RF pulses. However, when using black blood MRI sequences, the arterial wall is clearly visible (see figure 2) and also contrast within the arterial wall (e.g. in plaques) is much more apparent. Black blood MRI sequences may therefore be more appropriate to evaluate local strain or stiffness data of atherosclerotic carotid artery plaques.

To evaluate local strain information, image registration techniques can be used to find the deformation between MR images recorded at diastolic and systolic blood pressure. Non-rigid transformations and morphing methods based on image features like calcium spots or carotid bifurcations are commonly used to match images from different modalities (Nanayakkara et al., 2009). These techniques can also be used to match systolic and diastolic MR magnitude images of carotid artery plaques. The required deformations between both states could potentially be used to extract local strain information. In the following section, a new method is proposed to determine artery strain using non-rigid image registration between MR black blood images at different pressures.

Novel black blood analysis approach using non rigid registration 
The proposed method uses the deformations determined from non-rigid registration between MR black blood magnitude images, acquired using a cine PC MRI sequence with a low velocity encoding (VENC 1-5 $\mathrm{cm} / \mathrm{s}$ ). For the non-rigid image registration, we use Elastix, which is open-source software that was specifically designed to determine deformation data between two (medical) images, developed by Klein and Staring at the Image Science Institute at the University Medical Center Utrecht, the Netherlands (Klein et al., 2010). The accuracy and precision of the registration strongly depends on the signal-to-noise ratio, the resolution and the contrast of the images. Using grayscale images of idealized plaque models, Elastix was evaluated for different image resolutions and signal-to-noise ratios (SNR) (figure 5). A fixed translation of $5 \mathrm{~mm}$ (resolution test) or 5 pixels (SNR test) was applied to the images. The relative translation error between the applied translation and the detected translation by Elastix was less than $1.5 \%$ for all resolutions, and about $0.5 \%$ for the highest resolutions. Above a SNR of 5 , the absolute error was less than 0.5 pixels, with a strong increase in error for the images with a SNR below 5 . The final result and confidence interval of the registration thus strongly depend on the image resolution and the SNR of the MRI scan, and should therefore be carefully evaluated for each type of image.

The black blood PC MRI images (SNR 25) from figure 2 are used for non-rigid image registration. The smallest and largest carotid cross-section are selected and used as fixed and moving image (see figure 6). A non-rigid B-spline registration method was used and the optimal grid size was determined to be 9 pixels. A finer grid size is able to measure more local deformations, while the results contain more noise; a coarser grid size gives more averaged results without being able to capture variations in strain in the vessel wall.

In figure 6, manually drawn masks were used to visualize the region of interest where the displacement between the fixed and moving images is determined. The displacement field in $\mathrm{x}$-direction clearly shows that the left side of the wall moves to the left (negative $x$-displacement), while the right side moves to 
the right (positive $\mathrm{x}$-displacement). To determine the principal stretches in the region of interest, the deformation tensor $\mathbf{F}$ of the registration is determined by computing the spatial derivative of the $\mathbf{x}$-and y-deformations. Thereafter, the right Cauchy-Green deformation tensor $\mathbf{C}=\mathbf{F}^{\mathbf{\top}} \mathbf{F}$ is computed. The two eigenvalues of tensor $\mathbf{C}$ per pixel in the image are the squares of the maximum and minimum principal stretches, which are shown in figure 6 for our example. Maximum stretch values in this example were 1.05 on average (range: $1.01-1.07$, Cauchy strains between $1 \%$ and $7 \%$ ), while minimum principal stretch ranged from $0.97-1.02$ (Cauchy strains between $-3 \%$ to $+2 \%$ ) in the presented example. Visual inspection showed that the direction of the maximum stretch generally corresponded to the circumferential direction, while the minimum stretch was more oriented radially (data not shown). Maximum and minimum principle stretches are quite homogeneous over the vessel wall, which was expected for our healthy volunteer. A more heterogeneous stretch distribution may be expected in the presence of an atherosclerotic plaque. The proposed method seems to be suitable for local strain analysis for atherosclerotic plaque, but is also limited by the acquired MRI data. The method will benefit from efforts to increase the spatial and temporal resolution, as well as improvements in contrast and SNR of the MRI data. 


\section{Discussion and future perspectives}

In this review, several methods have been described that are able to derive strain or stiffness information of carotid arteries from MRI data. In theory, vessel wall strain can be obtained either by direct MRI measurements using displacement, velocity, or strain encoding, or obtained from postprocessing techniques, using bright or black blood cine MRI data.

In general, the current technical limitations related to the spatial and temporal resolution of MRI prohibit the use of MR strain imaging techniques that were developed for the heart and the aorta, to be applied to the carotid arteries. For cine MRI, the temporal resolution is inversely proportional to the repetition time (TR). However, TR cannot be lowered at will to increase the temporal resolution, because of gradient hardware limitations. The use of dedicated receiver coils (Balu et al., 2009) and stronger gradient systems (McNab et al., 2013) will result in higher spatial and temporal resolutions and may pave the way for more accurate strain imaging in the (near) future. Developments on higher field strength clinical MRI scanners may also add to this, although higher magnetic fields mainly increase the SNR, and not the temporal or spatial resolution in cine MRI (Koning et al., 2013; Yarnykh et al., 2006). Several techniques have been developed to increase the temporal resolution of cine MRI, e.g. view sharing that effectively increases the number of sample points throughout the heart cycle by performing temporal interpolation (Markl and Hennig, 2001; Slavin and Bluemke, 2005). Presently, more innovative techniques to increase the temporal resolution are being proposed in the literature, like shared velocity encoding (Lin et al., 2012) and retrospective self-gating (Coolen et al., 2013). Unfortunately, these techniques are not yet commercially available. With the current clinically available MRI scanners and imaging techniques, carotid artery plaque strain imaging remains very challenging.

Post-processing techniques based on cine MRI data may serve as a good alternative to determine strain in carotid artery plaques. The effectiveness of post-processing methods based on black blood cine MRI 
images is mainly determined by the spatial resolution of the MRI scan and the contrast in the scan. The black blood cine PC MRI sequence used in this paper and the black blood cine approach put forward by Koktzoglou et al. (2013) both seem suitable for performing strain analysis, although improvements in cine MRI are essential, especially in the temporal resolution (Koktzoglou, 2013).

Non-rigid registration methods may be used to determine the local deformation field of carotid artery plaques from cine MRI. In the example in figure 6, we present the stretch values of a healthy carotid artery as determined with B-spline non-rigid image registration. This is the first time that both in-plane principle stretch values are determined from in vivo MRI data of a human carotid artery. Although the presented B-spline registration technique can provide sub-pixel accuracy to register MRI images, it highly depends on the image quality (resolution, contrast, and SNR). Also, the used B-spline parameters may influence the result of the registration. Depending on the size of the structure of interest, a finer or coarser grid for the B-splines may be required to detect local tissue stretch.

A limitation of the presented techniques based on 2D bright and black blood MR images is out-of-plane motion. It was shown before that carotid arteries undergo a significant amount of longitudinal motion (Cinthio et al., 2006). Depending on the MRI slice thickness this may result in different parts of the carotid artery being imaged at different phases in the cardiac cycle. This longitudinal motion may have a large effect when imaging carotid artery plaques. 3D time resolved MRI imaging protocols may in the future provide 3D image registration data that account for this longitudinal motion. 


\section{Conclusions}

In this review, current MR techniques and post-processing techniques that may be used to evaluate carotid artery vessel wall and plaque strain are discussed. Although most MR techniques suffer from relative low spatial and temporal resolution, future developments in DENSE MRI or advanced cine PC MRI sequences may provide accurate displacement or velocity information. Improvements in MRI hardware should be focused mainly on faster gradient systems to improve the temporal resolution. Postprocessing techniques based on black blood cine MRI, like the one proposed in this review, may provide a good estimate of plaque strain or mechanical properties, but are also dependent on the spatial and temporal resolution of the MRI scan. However, they seem to be the most promising approach for MRI based plaque strain analysis in the near future. 


\section{References}

Akyildiz, A.C., Speelman, L., Van Brummelen, H., Gutiérrez, M.A., Virmani, R., Van Der Lugt, A., Van Der Steen, A.F., Wentzel, J.J., Gijsen, F.J., 2011. Effects of intima stiffness and plaque morphology on peak cap stress. Biomed. Eng. Online 10, 25.

Aletras, A.H., Ding, S., Balaban, R.S., Wen, H., 1999. DENSE: displacement encoding with stimulated echoes in cardiac functional MRI. J. Magn. Reson. 137, 247-52.

Avril, S., Schneider, F., Boissier, C., 2011a. In vivo time-resolved sub-pixel measurements of wall deformation in the common carotid artery. In: 7th European Symposium of Vascular Biomaterials, ESVB 2011. pp. 1-35.

Avril, S., Schneider, F., Boissier, C., Li, Z.-Y., 2011b. In vivo velocity vector imaging and time-resolved strain rate measurements in the wall of blood vessels using MRI. J. Biomech. 44, 979-83.

Balu, N., Yarnykh, V.L., Scholnick, J., Chu, B., Yuan, C., Hayes, C., 2009. Improvements in carotid plaque imaging using a new eight-element phased array coil at 3T. J. Magn. Reson. Imaging 30, 1209-14.

Beauchemin, S.S., Barron, J.L., 1995. The computation of optical flow. ACM Comput. Surv. 27, 433-466.

Bernstein, M.A., King, K.F., Joe Zhou, X., 2004. Handbook of MRI pulse sequences, Pulse. Elsevier Academic Press.

Bohte, A.E., Garteiser, P., De Niet, A., Groot, P.F.C., Sinkus, R., Stoker, J., Nederveen, A.J., 2013. MR Elastography of the Liver: Defining Thresholds for Detecting Viscoelastic Changes. Radiology.

Casscells, W., Naghavi, M., Willerson, J.T., 2003. Vulnerable atherosclerotic plaque: a multifocal disease. Circulation 107, 2072-5.

Cinthio, M., Ahlgren, A.R., Bergkvist, J., Jansson, T., Persson, H.W., Lindström, K., 2006. Longitudinal movements and resulting shear strain of the arterial wall. Am. J. Physiol. Heart Circ. Physiol. 291, H394-402.

Coolen, B.F., Abdurrachim, D., Motaal, A. G., Nicolay, K., Prompers, J.J., Strijkers, G.J., 2013. High frame rate retrospectively triggered Cine MRI for assessment of murine diastolic function. Magn. Reson. Med. 69, 648-56.

D’Agostino, R.B., Grundy, S., Sullivan, L. M., Wilson, P., 2001. Validation of the Framingham coronary heart disease prediction scores: results of a multiple ethnic groups investigation. JAMA 286, 180-7.

Draney, M.T., Arko, F.R., Alley, M.T., Markl, M., Herfkens, R.J., Pelc, N.J., Zarins, C.K., Taylor, C. a, 2004. Quantification of vessel wall motion and cyclic strain using cine phase contrast MRI: in vivo validation in the porcine aorta. Magn. Reson. Med. 52, 286-95. 
Draney, M.T., Herfkens, R.J., Hughes, T.J.R., Pelc, N.J., Wedding, K.L., Zarins, C.K., Taylor, C. a., 2002. Quantification of Vessel Wall Cyclic Strain Using Cine Phase Contrast Magnetic Resonance Imaging. Ann. Biomed. Eng. 30, 1033-1045.

Franquet, A., Avril, S., Le Riche, R., Badel, P., Schneider, F., Li, Z., Boissier, C., Favre, J., 2013a. A new method for the in vivo identification of mechanical properties in arteries from cine MRI images: theoretical framework and validation. IEEE Trans. Med. Imaging 6, 1-16.

Franquet, A., Avril, S., Le Riche, R., Badel, P., Schneider, F. C., Boissier, C., Favre, J.-P., 2013b. Identification of the in vivo elastic properties of common carotid arteries from MRI: A study on subjects with and without atherosclerosis. J. Mech. Behav. Biomed. Mater. 27, 184-203.

Golledge, J., Greenhalgh, R.M., Davies, A.H., 2000. The symptomatic carotid plaque. Stroke. 31, 774-81.

Halliday, A., Mansfield, A., Marro, J., Peto, C., Peto, R., Potter, J., Thomas, D., 2004. Prevention of disabling and fatal strokes by successful carotid endarterectomy in patients with out recent neurological symptoms: randomised controlled trial., Lancet.

Hamdan, A., Thouet, T., Kelle, S., Wellnhofer, E., Paetsch, I., Gebker, R., Schnackenburg, B., Fahmy, A.S., Osman, N.F., Bornstedt, A., Fleck, E., 2009. Strain-encoded MRI to evaluate normal left ventricular function and timing of contraction at 3.0 Tesla. J. Magn. Reson. Imaging 29, 799-808.

Hansen, H.H.G., Lopata, R.G.P., de Korte, C.L., 2009. Noninvasive carotid strain imaging using angular compounding at large beam steered angles: validation in vessel phantoms. IEEE Trans. Med. Imaging 28, 872-80.

Herment, A., Lefort, M., Kachenoura, N., De Cesare, A., Taviani, V., Graves, M.J., Pellot-Barakat, C., Frouin, F., Mousseaux, E., 2011. Automated estimation of aortic strain from steady-state freeprecession and phase contrast MR images. Magn. Reson. Med. 65, 986-93.

Huwart, L., Sempoux, C., Vicaut, E., Salameh, N., Annet, L., Danse, E., Peeters, F., ter Beek, L.C., Rahier, J., Sinkus, R., Horsmans, Y., Van Beers, B.E., 2008. Magnetic resonance elastography for the noninvasive staging of liver fibrosis. Gastroenterology 135, 32-40.

Klein, S., Staring, M., Murphy, K., Viergever, M.A., Pluim, J.P.W., 2010. elastix: a toolbox for intensitybased medical image registration. IEEE Trans. Med. Imaging 29, 196-205.

Koktzoglou, I., 2013. 4D Dark blood arterial wall magnet ic resonance imaging: methodology and demonstration in the carotid arteries. Magn. Reson. Med. 69, 956-65.

Kolipaka, A., Woodrum, D., Araoz, P. a., Ehman, R.L., 2011. MR elastography of the in vivo abdominal aorta: A feasibility study for comparing aortic stiffness between hypertensives and normotensives. J. Magn. Reson. Imaging 000, n/a-n/a.

Koning, W., Bluemink, J.J., Langenhuizen, E. a J., Raaijmakers, a J., Andreychenko, a, van den Berg, C. a T., Luijten, P.R., Zwanenburg, J.J.M., Klomp, D.W.J., 2013. High-resolution MRI of the carotid arteries 
using a leaky waveguide transmitter and a high-density receive array at 7 T. Magn. Reson. Med. 69, $1-8$.

Kuijer, J.P.A., Hofman, M.B.M., Zwanenburg, J.J.M., Marcus, J.T., van Rossum, A.C., Heethaar, R.M., 2006. DENSE and HARP: two views on the same technique of phase-based strain imaging. J. Magn. Reson. Imaging 24, 1432-8.

Lin, A.P., Bennett, E., Wisk, L.E., Gharib, M., Fraser, S.E., Wen, H., 2008. Circumferential strain in the wall of the common carotid artery: comparing displacement-encoded and cine MRI in volunteers. Magn. Reson. Med. 60, 8-13.

Lin, H., Bender, J.A., Ding, Y., Chung, Y., Hinton, A.M., Pennell, M.L., Whitehead, K.K., Raman, S. V, Simonetti, O.P., 2012. Shared velocity encoding: A method to improve the temporal resolution of phase-contrast velocity measurements. Magn. Reson. Med. 68, 703-10.

Lotz, J., Meier, C., Leppert, A., Galanski, M., 2002. Cardiovascular flow measurement with phase-contrast MR imaging: basic facts and implementation. Radiographics 22, 651-71.

Makris, G.C., Nicolaides, A.N., Xu, X.Y., Geroulakos, G., 2010. Introduction to the biomechanics of carotid plaque pathogenesis and rupture: review of the clinical evidence. Br. J. Radiol. 83, 729-35.

Markl, M., Hennig, J., 2001. Phase contrast MRI with improved temporal resolution by view sharing: kspace related velocity mapping properties. Magn. Reson. Imaging 19, 669-76.

McNab, J.A., Edlow, B.L., Witzel, T., Huang, S.Y., Bhat, H., Heberlein, K., Feiweier, T., Liu, K., Keil, B., Cohen-Adad, J., Tisdall, M.D., Folkerth, R.D., Kinney, H.C., Wald, L.L., 2013. The Human Connectome Project and beyond: Initial applications of $300 \mathrm{mT} / \mathrm{m}$ gradients. Neuroimage.

Merkx, M.A.G., Van T Veer, M., Speelman, L., Breeuwer, M., Buth, J., Van De Vosse, F.N., 2009. Importance of initial stress for abdominal aortic aneurysm wall motion: dynamic MRI validated finite element analysis. J. Biomech. 42, 2369-2373.

Naim, C., Cloutier, G., Mercure, E., Destrempes, F., Qin, Z., El-Abyad, W., Lanthier, S., Giroux, M.-F., Soulez, G., 2013. Characterisation of carotid plaques with ultrasound elastography: feasibility and correlation with high-resolution magnetic resonance imaging. Eur. Radiol. 23, 2030-41.

Nanayakkara, N.D., Chiu, B., Samani, A., Spence, J.D., Samarabandu, J., Parraga, G., Fenster, A., 2009. Nonrigid registration of three-dimensional ultrasound and magnetic resonance images of the carotid arteries. Med. Phys. 36, 373-85.

Nguyen, T.D., de Rochefort, L., Spincemaille, P., Cham, M.D., Weinsaft, J.W., Prince, M.R., Wang, Y., 2008. Effective motion-sensitizing magnetization preparation for black blood magnetic resonance imaging of the heart. J. Magn. Reson. Imaging 28, 1092-100.

Osman, N.F., 2003. Detecting stiff masses using strain-encoded (SENC) imaging. Magn. Reson. Med. 49, 605-8. 
Sadat, U., Teng, Z., Young, V.E., Graves, M.J., Gaunt, M.E., Gillard, J.H., 2011. High-resolution magnetic resonance imaging-based biomechanical stress analysis of carotid atheroma: a comparison of single transient ischaemic attack, recurrent transient ischaemic attacks, non-disabling stroke and asymptomatic patient groups. Eur. J. Vasc. Endovasc. Surg. 41, 83-90.

Schaar, J.A., De Korte, C.L., Mastik, F., Strijder, C., Pasterkamp, G., Boersma, E., Serruys, P.W., Van Der Steen, A.F.W., 2003. Characterizing vulnerable plaque features with intravascular elastography. Circulation 108, 2636-41.

Schaar, J.A., van der Steen, A.F.W., Mastik, F., Baldewsing, R.A., Serruys, P.W., 2006. Intravascular palpography for vulnerable plaque assessment. J. Am. Coll. Cardiol. 47, C86-91.

Slager, C.J., Wentzel, J.J., Gijsen, F.J.H., Schuurbiers, J.C.H., van der Wal, A.C., van der Steen, A.F.W., Serruys, P.W., 2005. The role of shear stress in the generation of rupture-prone vulnerable plaques. Nat. Clin. Pract. Cardiovasc. Med. 2, 401-7.

Slavin, G.S., Bluemke, D.A., 2005. Spatial and temporal resolution in cardiovascular MR imaging: review and recommendations. Radiology 234, 330-8.

Tee, M., Noble, J.A., Bluemke, D.A., 2013. Imaging techniques for cardiac strain and deformation: comparison of echocardiography, cardiac magnetic resonance and cardiac computed tomography. Expert Rev. Cardiovasc. Ther. 11, 221-31.

Van't Veer, M., Buth, J., Merkx, M., Tonino, P., van den Bosch, H., Pijls, N., van de Vosse, F., 2008. Biomechanical properties of abdominal aortic aneurysms assessed by simultaneously measured pressure and volume changes in humans. J. Vasc. Surg. 48, 1401-7.

Van Wijk, D.F., Strang, A.C., Duivenvoorden, R., Enklaar, D.-J.F., van der Geest, R.J., Kastelein, J.J.P., de Groot, E., Stroes, E.S.G., Nederveen, A.J., 2013. Increasing spatial resolution of 3T MRI scanning improves reproducibility of carotid arterial wall dimension measurements. MAGMA.

Wang, H., Amini, A.A., 2012. Cardiac motion and deformation recovery from MRI: a review. IEEE Trans. Med. Imaging 31, 487-503.

Wedding, K.L., Draney, M.T., Herfkens, R.J., Zarins, C.K., Taylor, C.A., Pelc, N.J., 2002. Measurement of Vessel Wall Strain Using Cine Phase Contrast MRI 428, 418-428.

Xu, L., Chen, J., Glaser, K.J., Yin, M., Rossman, P.J., Ehman, R.L., 2013. MR elastography of the human abdominal aorta: A preliminary study. J. Magn. Reson. Imaging 000, 1-5.

Yarnykh, V.L., Terashima, M., Hayes, C.E., Shimakawa, A., Takaya, N., Nguyen, P.K., Brittain, J.H., McConnell, M. V, Yuan, C., 2006. Multicontrast black-blood MRI of carotid arteries: comparison between 1.5 and 3 tesla magnetic field strengths. J. Magn. Reson. Imaging 23, 691-8.

Young, A.A., Axel, L., Dougherty, L., Bogen, D.K., Parenteau, C.S., 1993. Validation of tagging with MR imaging to estimate material deformation. Radiology $188,101-8$. 
Table 1: Comparison of different MRI techniques for measuring strain and/or stiffness in the carotid artery. Spatial and temporal resolutions indicate what is presented in literature.

\begin{tabular}{|c|c|c|c|c|c|c|c|}
\hline Technique & $\begin{array}{l}\text { Inner } \\
\text { vessel } \\
\text { wall }\end{array}$ & $\begin{array}{l}\text { Outer } \\
\text { vessel } \\
\text { wall }\end{array}$ & $\begin{array}{l}\text { Temporal } \\
\text { resolution } \\
\text { (ms) }\end{array}$ & $\begin{array}{l}\text { Spatial } \\
\text { resolution } \\
(\mathrm{mm})\end{array}$ & Disadvantages & Advantages & Used in carotids \\
\hline Cine MRI & + & - & $20-50$ & 0.6 & $\begin{array}{l}\text { No vessel wall } \\
\text { contrast }\end{array}$ & Widely available & $\begin{array}{l}\text { (Avril et al., 2011a, } \\
\text { 2011b; Franquet et } \\
\text { al., 2013a, 2013b) }\end{array}$ \\
\hline $\begin{array}{l}\text { Black blood } \\
\text { cine MRI }\end{array}$ & + & + & 60 & $0.8(3 \mathrm{D})$ & Not yet available & $\begin{array}{l}\text { Volumetric and } \\
\text { isotropic coverage }\end{array}$ & (Koktzoglou, 2013) \\
\hline Cine PC MRI & + & + & $50-60$ & $0.3-0.8$ & $\begin{array}{l}\text { Impossible to } \\
\text { meet spatial and } \\
\text { temporal } \\
\text { requirements in } \\
\text { one sequence }\end{array}$ & Widely available & - \\
\hline DENSE & + & + & n.a. & 0.6 & $\begin{array}{l}\text { Additional MR } \\
\text { expertise required }\end{array}$ & $\begin{array}{l}\text { Displacement is } \\
\text { measured directly }\end{array}$ & (Lin et al., 2008) \\
\hline Elastography & + & - & n.a. & n.a. & $\begin{array}{l}\text { Additional MR } \\
\text { expertise and } \\
\text { hardware } \\
\text { required }\end{array}$ & $\begin{array}{l}\text { Stiffness directly } \\
\text { derived from MR } \\
\text { data }\end{array}$ & - \\
\hline
\end{tabular}



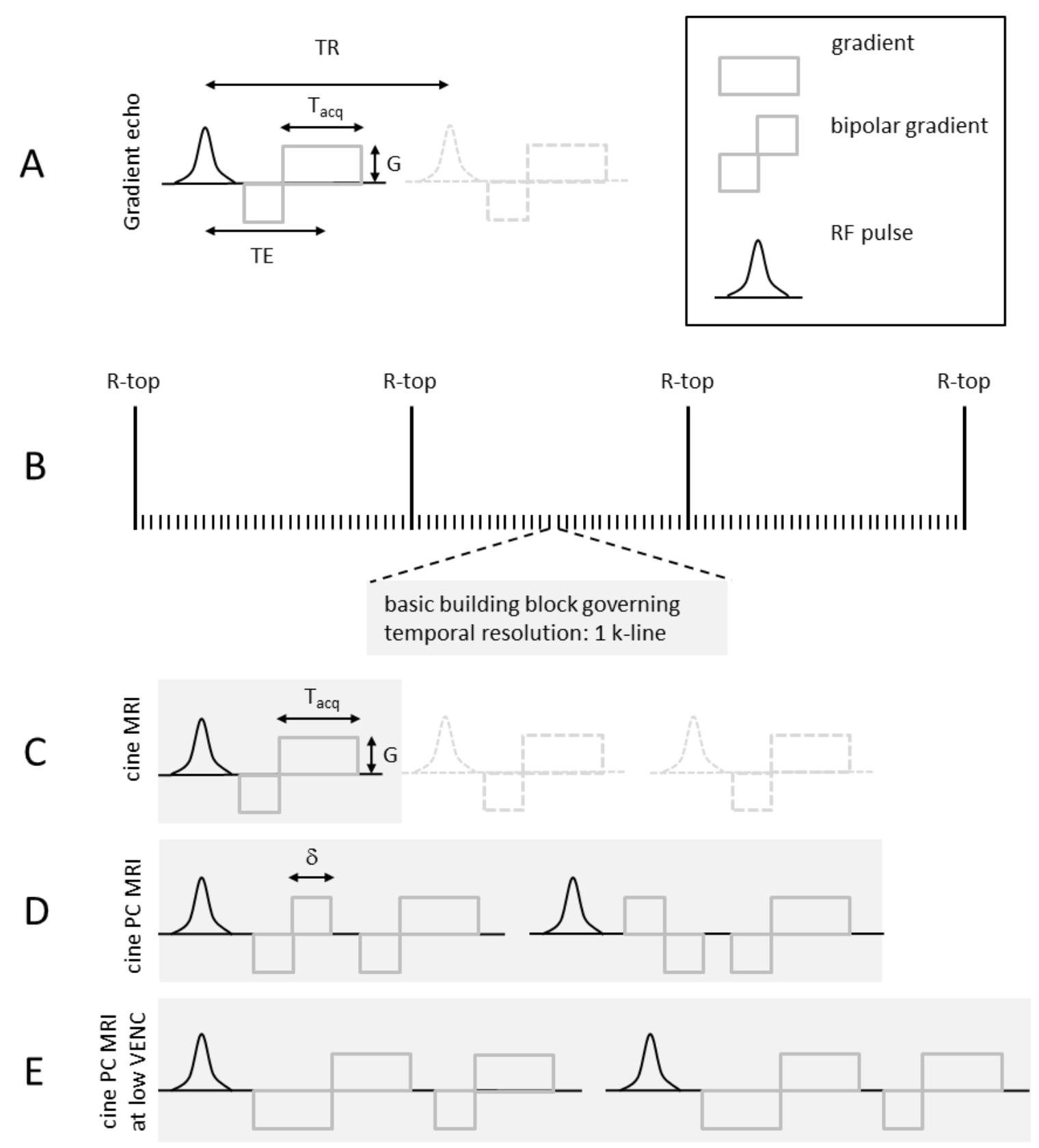

Figure 1: Illustration of several basic components of cine MRI. (A) The basic building block is constituted by a gradient echo sequence: a combination of a RF pulse and a readout gradient, with magnitude $G$. TE is the echo time, $T R$ repetition time, and $\mathrm{T}_{\text {acq }}$ the readout time. (B) During cine MRI, this gradient echo block is repeated many times during the heart cycle, filling $1 \mathrm{k}$-line for each RF pulse. (C) In basic cine MRI, the basic building block represents a standard gradient echo, which is synchronized with the heartbeat and images over the entire heart cycle are obtained. (D) In cine PC MRI, a bipolar gradient is added and two TRs are required for velocity encoding. (E) For low VENC values, the gradient duration is prolonged and the temporal resolution is even further reduced. 


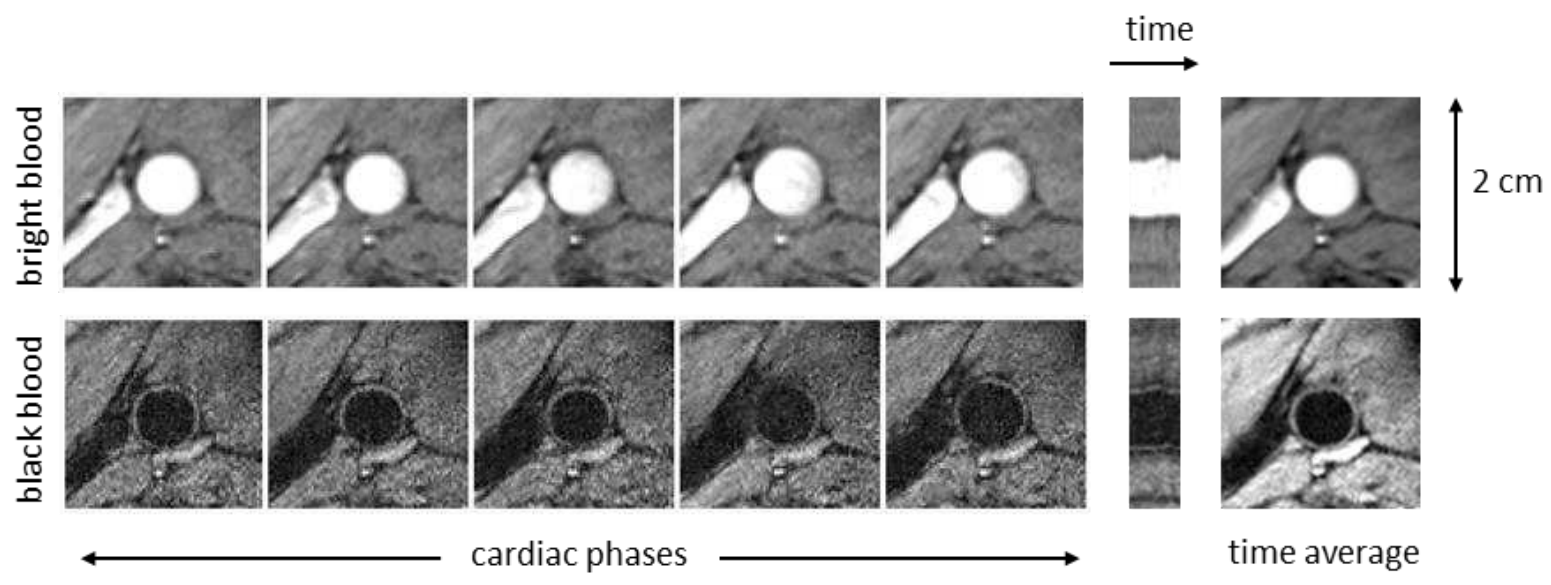

Figure 2: Bright blood (top) and black blood (bottom) images showing the common carotid artery, using cine PC MRI with a VENC of $100 \mathrm{~cm} / \mathrm{s}$ (bright blood) and $1 \mathrm{~cm} / \mathrm{s}$ (black blood). The temporal resolution for bright blood is $17 \mathrm{~ms}$ (TR/TE 8.5/3.3 ms, FA 10 degrees) and for black blood $50 \mathrm{~ms}$ (TR/TE 25/13 ms, FA 10 degrees), with a non-interpolated spatial resolution $0.3 \mathrm{~mm}$ (pixel size) and a slice thickness of 5 $\mathrm{mm}$. A saturation slab caudal to the imaging plane was added to improve the inherent blood suppression from the black blood cine PC MRI sequence. A reformatted slice in the temporal direction of the imaging stack illustrates the pulsatile motion of the vessel wall in time. The time average image at the right displays the average image of all cine images. 

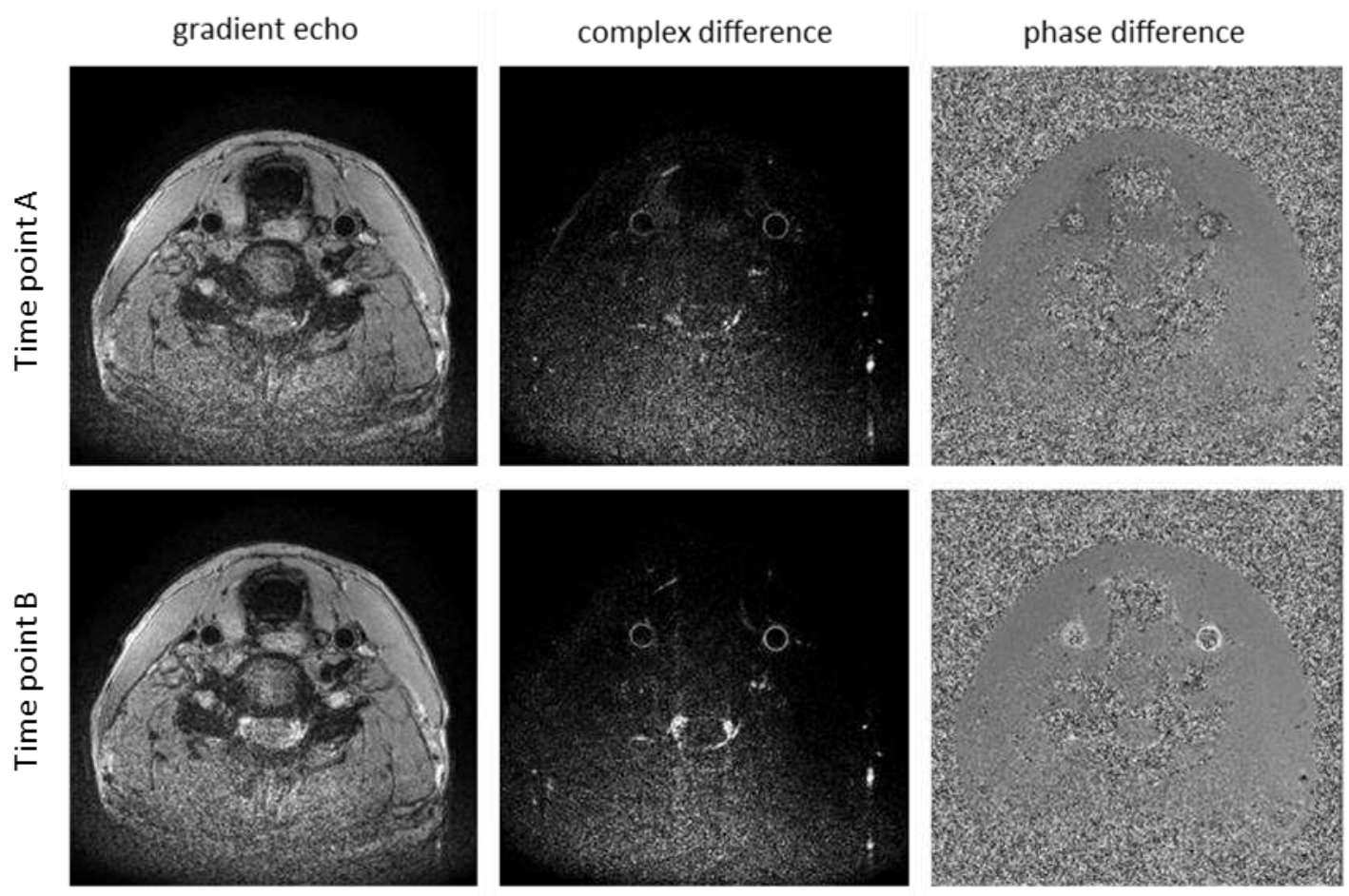

Figure 3: Black blood cine PC MRI of the common carotid artery at two time points in the cardiac cycle. (Left) Gradient echo magnitude images. (Middle) Complex difference images showing the magnitude of difference between the bipolar toggles. (Right) Phase difference images representing motion in caudalcranial direction. VENC $1 \mathrm{~cm} / \mathrm{s}$, temporal resolution $56 \mathrm{~ms}$ (TR/TE 28/12.5 ms, FA 10 degrees), noninterpolated spatial resolution $0.4 \mathrm{~mm}$, slice thickness of $4 \mathrm{~mm}$. A saturation slab caudal to the imaging plane was added to improve the inherent blood suppression from the black blood cine PC MRI sequence. At time point A (top row), negative values at the arterial wall can be seen in the phase difference image, representing downward motion. At time point B (bottom row) positive values in the arterial wall represent an upward motion of the vessel. 


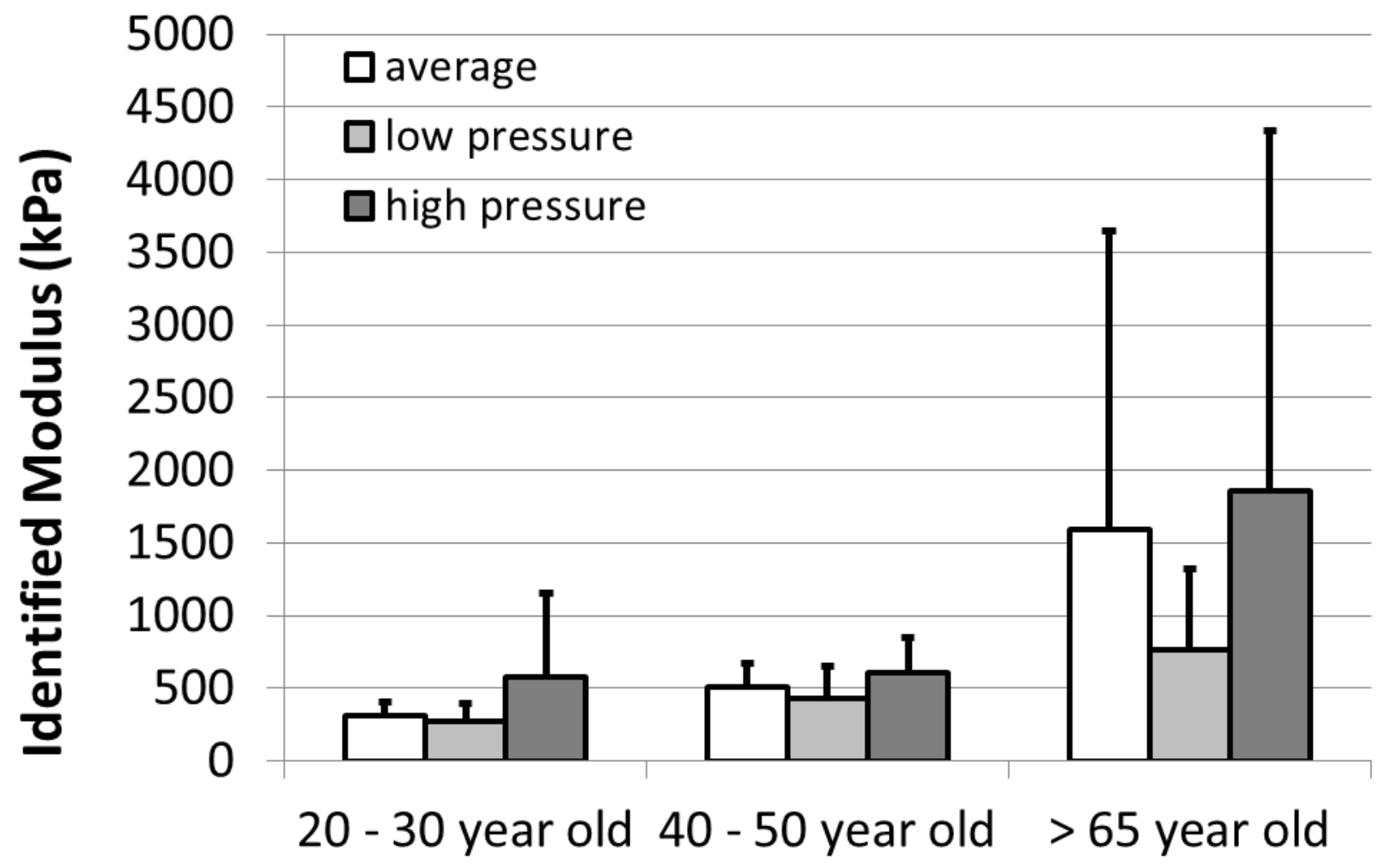

Figure 4: Global elastic modulus of the common carotid artery wall identified by Franquet et al. (2013b) for low and high blood pressure and averaged over the complete cardiac cycle for different age groups. Figure adapted from Franquet et al., 2013b. 


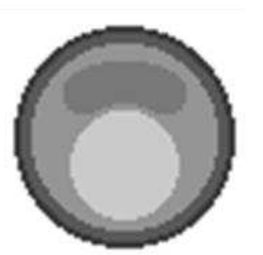

$64 \times 64$

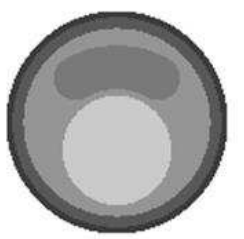

$320 \times 320$

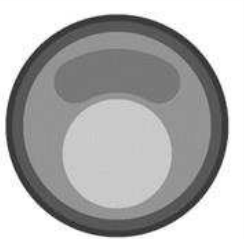

$512 \times 512$

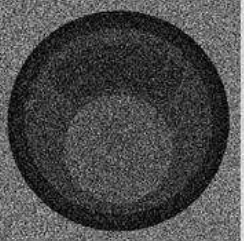

SNR 1.2

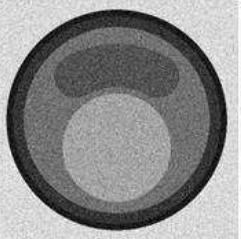

SNR 5

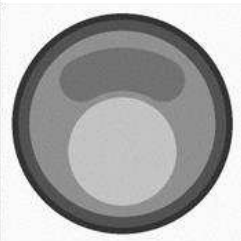

SNR 35

Translation Error

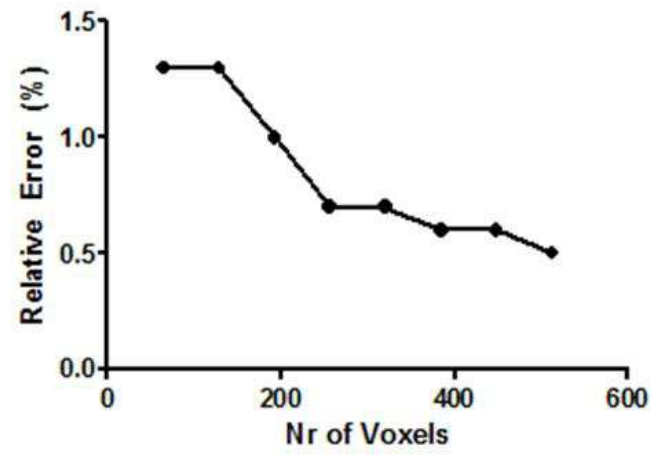

Translation Error

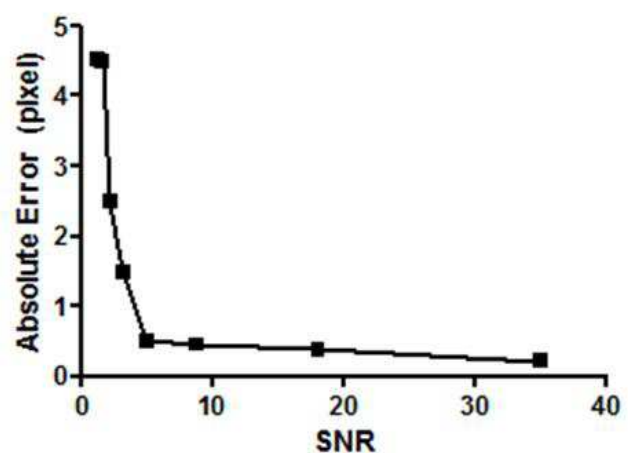

Figure 5: Translation error after non-rigid image registration of an idealized plaque model with a $5 \mathrm{~mm}$ translation as function of the image resolution (left) and of an idealized plaque model with 5 pixels translation as function of the image signal-to-noise ratio (right). 


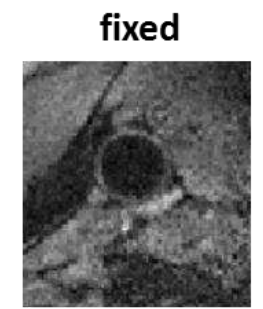

$\mathrm{x}$ displacement

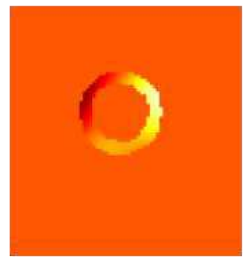

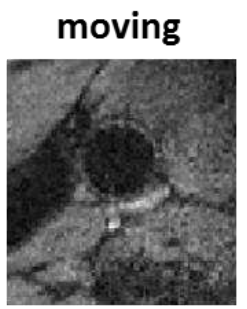

y displacement

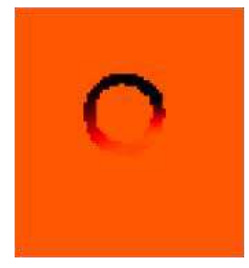

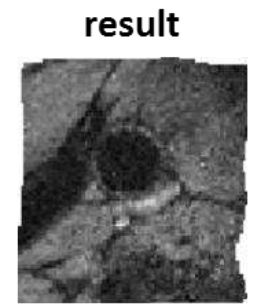

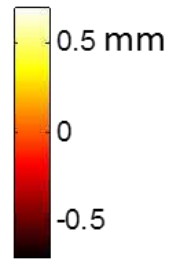

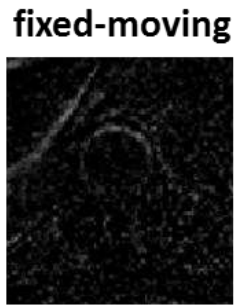

fixed-result

Max. Princ. stretch Min. Princ. stretch

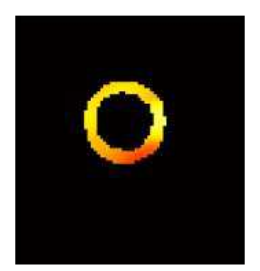

Figure 6: Fixed (diastolic), moving (systolic) images and B-spline non-rigid deformation result, with the subtraction between fixed and moving images showing the edge of the vessel, which disappears in the fixed and result subtraction (top row). The displacement fields in $x$ - and $y$-direction are used to determine maximum and minimum principal stretch values in the carotid wall (bottom row). 